\title{
The formation of cube volumes in cold rolled fcc metals
}

\author{
C.S. LEE, R.E. SMALLMAN and B.J. DUGGAN* \\ School of Metallurgy and Materials, University of Birmingham, Birmingham B15 2TT, U.K. \\ ${ }^{*}$ Mechanical Engineering Department, University of Hong Kong, Pokfulam Road, Hong Kong
}

\begin{abstract}
It has recently been reported that in cold rolled copper, cube-oriented volumes are frequently found in long thin bands next to bands whose orientation is related to the cube orientation by a $30-40^{\circ}$ rotation about a <111> common pole. This microstructure would suggest that during recrystallisation, cube in the deformed structure can growth easily into its neighbour due to the special misorientation which allows a high boundary migration rate. The formation of this special microstructural arrangement is studied here by a generalised deformation banding model.
\end{abstract}

\section{Introduction}

Because of its simplicity and extreme sharpness, the cube annealing texture has been used as a major test area for recrystallisation theories. The alternative theories which exist, namely the orientated Nucleation (ON) and Orientated Growth (OG) theory, have both found support from a wide range of experimental data. This controversial situation can partly be explained by a recent observation [1] showing that in fact both the ON and OG condition are important. It was observed that in heavily cold rolled copper, about $30 \%$ of the cube volumes have a $\sim 30-40^{\circ}\langle 111\rangle$ rotation relationship with their immediate neighbours. Upon annealing, the cube nuclei grow only into the <111> rotated neighbours. The existence of cube volume supports the oN theory; their growth into the $\langle 111\rangle$ rotated neighbours supports the OG theory. While this work reconciles the two theories, the origin of the cube volume together with its $\left.30-40^{\circ}<111\right\rangle$ rotated neighbours is not yet understood. The formation of this special microstructural arrangement is studied by a generalised deformation banding model in this paper.

\section{Simulation Models and Results}

Deformation banding has been classified into two types [2]. In the first type, different regions of a grain deform with the same homogeneous strain but by using different slip systems resulting in bands of different orientations. In the second type, different regions of a grain deform with different strains but having an average strain equal to the externally imposed macroscopic strain.

The formation of cube volume by the first type of banding has been studied by Dillamore and Katoh (DK model) [3]. However, no follow-up work has been done on the orientations of the neighbours of the cube volume. on the other hand, a deformation model corresponding to the second type has recently been formulated by lee et al. [4-5] (DB model). It is the 
purpose of this paper to study the formation of cube volume and the orientations of its surrounding by the two types of deformation banding.

\section{A. The DB Model}

It has been shown experimentally that deformation banding forms in a three dimensional manner [4-5]. However, due to the complexity of a 3D model, only a one dimensional model is considered by assuming that banding take place in only one geometry. The choice of this geometry is made according to the observation [1] that thin layers of cube-oriented material having a special orientation relationship with their neighbours are parallel to the rolling plane. Hence, of the six geometrically possible modes (fig. 1), only mode IV and mode VI are considered. of which only the results from mode VI banding are reported as it was found that cube volumes are not produced by mode IV banding.

100 grains with random orientations were used as the initial material in the rolling texture simulation. After $40 \%$ reduction, deformation banding forms in about half of the grains. Two of the banded grains give near cube orientation with $\left.-15-20^{\circ}<111\right\rangle$ rotated neighbours. As strain further increases, more grains are banded. At $64 \%$ reduction, four of the grains banded to give near cube orientations; of which, two have neighbouring bands whose orientations can be described by a $\left.-30-40^{\circ}<111\right\rangle$ rotation relationship with the cube orientation.

After $80 \%$ rolling, four of these grains have near cube orientations together with $-30-40^{\circ}<111>$ rotated neighbours (fig. 2 ). The total volume fraction of cube-oriented material is estimated to be $2.3 \%$. As the strain increases further, the volume fraction of cube material remains fairly constant. However, there is an increase in the misorientation between the cube and their neighbouring bands. At $87 \%$ reduction, three grains give the near cube bands; one has a $\sim 30^{\circ}\langle 111\rangle$ rotated neighbour and the other two have $-50^{\circ}\langle 111\rangle$ rotated neighbours. At $92 \%$ reduction, near cube bands are found in three grains, one has a $\left.\sim 10^{\circ}<111\right\rangle$ neighbour and the other have $\left.\sim 60^{\circ}<111\right\rangle$ rotated (ie twin of cube) neighbours. The decay of the $30-40^{\circ}\langle 111\rangle$ rotated neighbours at high strains is considered to be due to a change in deformation mode and is discussed in detail in the next section.

\section{B. The DR Model}

The essential idea in the DK model is that only those parts of a grain which have an exact $\{010\}$ ND initial orientation would rotate to the cube orientation; any other region which has even the slightest deviation from $\{010\}$ ND would rotate towards other orientations. For example, if a grain of initial orientation $\{010\}<u v w>$ has an orientation gradient from $B$ though $A$ to $B$ ' as shown in figure 3. "A" would rotate towards the cube orientation while $B$ and $B$ ' rotate towards other orientations. Hence, the cube-oriented region will be sandwiched by materials with the final orientations of $B$ and $\mathrm{B}^{\prime}$. These final orientations are studied by using various initial orientations obtained by a $5^{\circ}$ rotation from $\{010\}<001>$ about RD followed by $5,10 \ldots 45^{\circ}$ rotations about ND as shown in figure 4 . After 92\% simulated rolling reduction, with respectively the FC (Full Constraint) and RC (Relaxed Constraint) Taylor models, grains 1 to 3 and 4 to 7 rotate to give orientations with $30-40^{\circ}\langle 111\rangle$ rotation relationship with the cube orientation.

\section{A Generalised Deformation Banding Model}

It is now clear that both the DB and DK mechanism can explain the formation of cube-oriented material surrounded by the $\left.30-40^{\circ}<111\right\rangle$ rotated neighbours. As the two models correspond to two types of deformation banding which are known to occur, it is reasonable to suggest that cube-oriented material is produced by both sources in cold rolling. 
The relative contribution from the two sources is considered by studying the strengths and weaknesses of the two mechanisms.

With the DK mechanism, cube volumes are produced only from materials with initial orientations exactly of $\{100\}\langle u v w\rangle$. Thus, cube is formed only from a narrow range of initial orientations. Furthermore, cube forms only at the specific transitional boundaries of regions which take different rotation paths (fig. 3). Hence the volume fraction of cubeoriented material produced by the DK model is expected to be small.

While the DB model predicts a reasonable volume fraction of cubeoriented material $(\sim 2 \%)$ from intermediate to high strains, the main problem is that the $\left.30-40^{\circ}<111\right\rangle$ rotated neighbour of cube decays after $87 \%$ reduction. One possible cause is due to a change in deformation mechanism resulting from a change in deformation band geometry as strain increases. It is considered that at high strains when the grain as well as the individual deformation band are long and thin, the RC model gives a better description of the deformation [6]. Another possible cause of the decay might arise from the one dimensional assumption of the model. As only one deformation banding mode, instead of the three operating simultaneously as observed, was considered, it is unlikely that the present simplified model can give all the fine details.

A generalised model for the formation of cube texture is proposed here. It is considered that the DB mechanism operates predominantly from low to intermediates strains. The important function of this mechanism is, however, that it acts as a funnel to bring a wider range of orientations into near cube orientations, surrounded by neighbours of $30-40^{\circ}\langle 111\rangle$ rotation. The DK mechanism (with RC model) then operates to take near cube-oriented material even closer to the cube orientation at high strains. As the $30-40^{\circ}<111>$ rotated neighbours are near the $\left.\{123\}<634\right\rangle$ orientation, which is a stable rolling texture component for the RC model, its special misorientation with the cube orientation remains as strain increases.

\section{Conclusions}

A unified deformation banding theory for the formation of cube-oriented volumes in cold rolled FCC material is proposed. This theory suggests that cube-oriented volumes are produced by the co-operation of two different types of deformation banding. The first type corresponds to the classical Dillamore and Katoh model; the second type corresponds to the new DB model proposed by Lee and co-workers. The formation of cubeoriented volumes surrounded by $30-40^{\circ}\langle 111\rangle$ rotated neighbours are predicted by the both types of banding. This special microstructure provides favourable conditions for the growth of cube-oriented grains during recrystallisation.

\section{Acknowledgement}

C.S. Lee gladly acknowledges the croucher Foundation for financial support in the form of a Fellowship.

\section{References}

1. B.J. Duggan, K. Lücke, G. Köhlhoff and C.S. Lee, Acta Metall. Mater. (accepted).

2. G.Y. Chin, Textures in Research and Practice, Berlin, p.236 (1969).

3. I.L. Dillamore and H. Katoh, Metal Sci., 8, 73 (1974).

4. C.S. Lee, B.J. Duggan, and R.E. Smallman, Materials Modelling: from Theory to Technology, oxford, p.79. (1991).

5. C.S. Lee and B.J. Duggan, Acta Metall. Mater. (accepted) .

6. C.S. Lee, R.E. Smallman and B.J. Duggan, Scripta Metall. Mater. (accepted). 


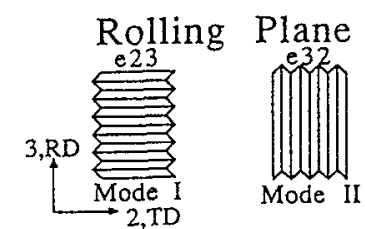

Longitudinal Section

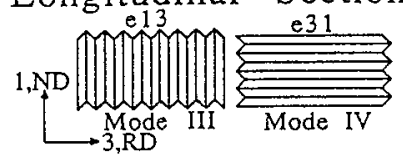

Transverse Section

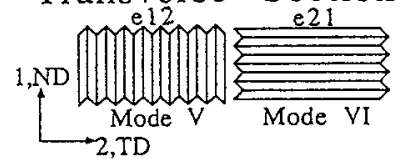

Figure 1. A schematic diagram showing six modes of deformation banding with respect to the rolling geometry.

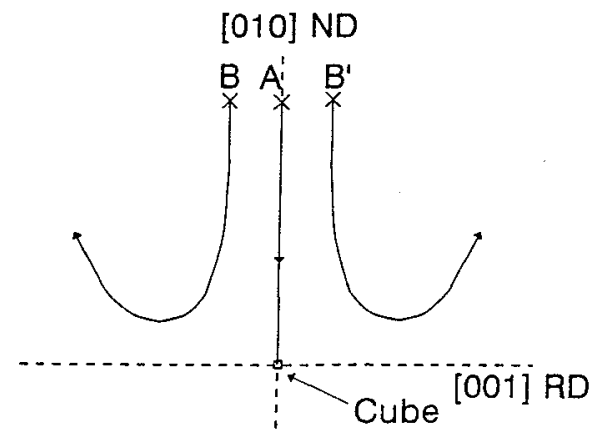

Figure 3. A schematic diagram showing how a grain of $\{010\}<u v w>$ (A) orientation give rise to a transition band with cube oriented materials.

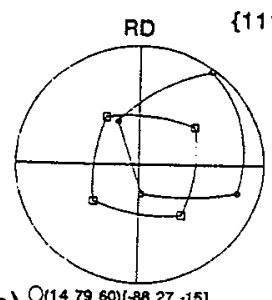

a) $\mathrm{O}_{147960)(8827 \cdot 151}$

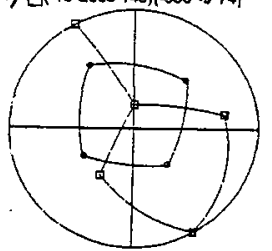

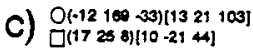

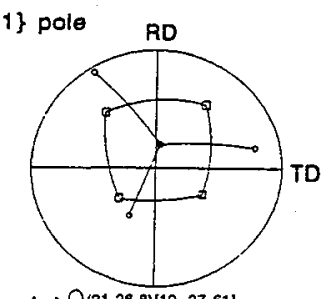

b)

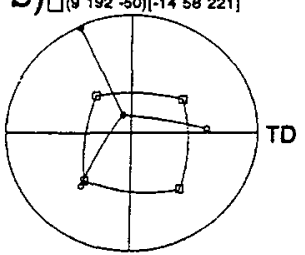

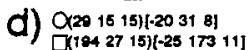

Figure 2. Poles figure showing orientations of the four grains which give near cube bands formed together with $\left.-30-40^{\circ} \quad<111\right\rangle$ rotated neighbours after $80 \%$ reduction with the $\mathrm{DB}$ model.

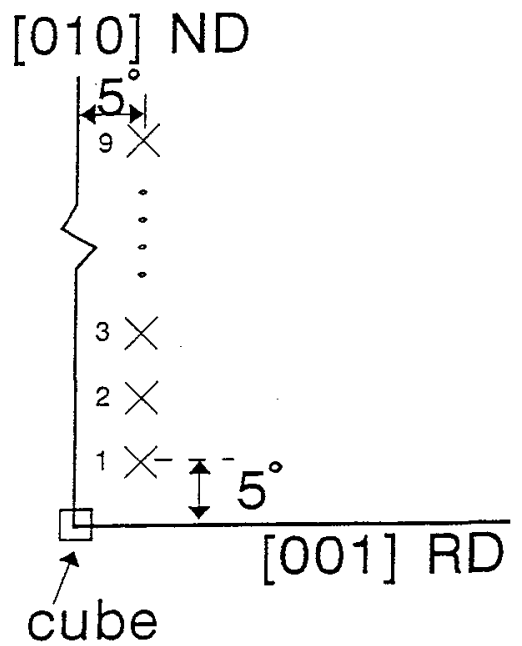

Figure 4. Initial orientations used for studying the possible cube surrounding of a cube transition band. 